\title{
INGESTÃO DE NUTRIENTES POR GESTANTES DA ATENÇÃO BÁSICA NO TOCANTINS
}

Nutrient intake by primary care pregnant women in Tocantins

Consumo de nutrientes por mujeres embarazadas de cuidado primario en Tocantins

“Artigo premiado no V Congresso Internacional de Atenção Primária à Saúde-V CIAPS, realizado em Teresina-PI, em 2021"

Karen Thayane de Oliveira Coqueiro • Mestranda em Ciências da Saúde pela UFT - Universidade Federal do Tocantins• E-mail: karen.coqueiro@gmail.com

Fabiana Cândida de Queiroz Santos Anjos • Docente do Curso de Medicina da UNIRG•Universidade de Gurupi - TO•E-mail: fcqsa@uol.com.br

Renata Junqueira Pereira • Docente do Curso de Nutrição da UFT • Universidade Federal do Tocantins E-mail: renatajunqueira@uft.edu.br

Autora correspondente:

Renata Junqueira Pereira E-mail: renatajunqueira@uft.edu.br 


\section{RESUMO}

Introdução: Em decorrência de alterações no comportamento alimentar materno durante os trimestres gestacionais, além das variações diárias e sazonais, podem ocorrer alterações na ingestão de nutrientes, que impactam diretamente no resultado obstétrico, sobretudo durante o primeiro trimestre de gestação. Objetivo: investigar o consumo de nutrientes por gestantes adultas, assistidas na atenção básica de Palmas, Tocantins, buscando identificar precocemente desvios evitáveis por meio de orientações dietéticas pré-natais. Metodologia: o estudo investigou 50 mulheres em quatro dos sete territórios de saúde de Palmas, Tocantins, por meio de um recordatório que investigou o consumo alimentar nas 24 horas anteriores à entrevista. Foram estimados pelo Dietbox, a ingestão diária de energia, carboidratos, proteínas, lipídios totais, ferro, calciferol, folato e cobalamina sem considerar os nutrientes obtidos por meio da suplementação medicamentos. Os dados foram analisados no programa Statistical Package for Social Sciences versão 23.0 por meio de estatísticas descritivas. Resultados: foram observados altos percentuais de inadequação da ingestão para todos os macros e micronutrientes estudados na população de gestantes. Destacaramse $82 \%$ das gestantes com ingestões inadequadas de energia; $98 \%$ de inadequação para o ácido fólico e $94 \%$ para ferro e cobalamina. A ingestão diária de calciferol esteve inadequada em 100\% das gestantes. Conclusão: o grupo de gestantes estudado está ingerindo nutrientes aquém do recomendado, estando o binômio mãe-filho sujeitos a resultados obstétricos indesejáveis, caso nenhuma intervenção seja realizada.

Palavras-Chave: gestação; consumo alimentar; micronutrientes.

\section{ABSTRACT}

Introduction: Due to changes in maternal eating behavior during the gestational trimesters, in addition to daily and seasonal variations, changes in nutrient intake may occur, which directly impact obstetric outcome, especially during the first trimester of pregnancy. Objective: to investigate the consumption of nutrients by adult pregnant women, assisted in primary care in Palmas, Tocantins, seeking to early identify preventable deviations through prenatal dietary guidelines. Methodology: the study investigated 50 women in four of the seven health territories in Palmas, through a recall that investigated food consumption in the 24 hours prior to the interview. The daily intake of energy, carbohydrates, proteins, total lipids, iron, calciferol, folate and cobalamin were estimated by Dietbox without considering the nutrients obtained through drug supplementation. Data were analyzed using the Statistical Package for Social Sciences version 23.0 using descriptive statistics. Results: high percentages of inadequate intake were observed for all macro and micronutrients studied in the population of pregnant women. $82 \%$ of pregnant women with inadequate energy take stood out; $98 \%$ unsuitable for folic acid and $94 \%$ for iron and cobalamin. The daily intake of calciferol was inadequate in $100 \%$ of the pregnant women. Conclusion: group of pregnant women studied is ingesting less than recommended nutrients, d the mother-child binomial is subject to undesirable obstetric results, if no ervention is performed.

words: gestation; food consumption; micronutrients. 


\section{RESUMEN}

Introducción: Debido a los cambios en la conducta alimentaria materna durante los trimestres gestacionales, además de las variaciones diarias y estacionales, pueden ocurrir cambios en la ingesta de nutrientes que impactan directamente en el resultado obstétrico, especialmente durante el primer trimestre del embarazo. Objetivo: investigar el consumo de nutrientes por parte de gestantes adultas, atendidas en atención primaria en Palmas, Tocantins, buscando identificar desviaciones precoces evitables a través de guías dietéticas prenatales. Metodología: el estudio investigó a 50 mujeres en cuatro de los siete territorios de salud en Palmas, a través de un recordatorio que investigó el consumo de alimentos en las 24 horas previas a la entrevista. Dietbox estimó la ingesta diaria de energía, carbohidratos, proteínas, lípidos totales, hierro, calciferol, folato y cobalamina sin considerar los nutrientes obtenidos a través de la suplementación farmacológica. Los datos se analizaron utilizando el paquete estadístico para ciencias sociales versión 23.0 utilizando estadística descriptiva. Resultados: se observaron altos porcentajes de ingesta inadecuada para todos los macro y micronutrientes estudiados en la población de gestantes. Se destacó el $82 \%$ de las embarazadas con aporte energético inadecuado; $98 \%$ inadecuado para ácido fólico y $94 \%$ para hierro y cobalamina. La ingesta diaria de calciferol fue inadecuada en el 100\% de las embarazadas. Conclusión: el grupo de gestantes estudiado está ingiriendo menos nutrientes de los recomendados, y el binomio madre-hijo está sujeto a resultados obstétricos indeseables, si no se realiza ninguna intervención.

Palabras clave: gestación; consumo de comida; micronutrientes. 


\section{Introdução}

Em decorrência de alterações no comportamento alimentar materno durante os trimestres gestacionais, além das variações diárias e sazonais, podem ocorrer alterações na ingestão de nutrientes, que impactam diretamente no resultado obstétrico ${ }^{1}$.

Paralelamente, a saúde do feto dependerá da condição nutricional prégestacional da mãe e, durante o primeiro trimestre de gestação, além das reservas de energia, macro e micronutrientes. Já no segundo e terceiro trimestres, as circunstâncias ambientais, como trabalho, estresse, alimentação, dentre outras, poderão influenciar de forma direta o estado nutricional materno e fetal ${ }^{2}$.

Assim, conhecer o estado nutricional dietético durante a gestação torna-se fundamental. A orientação dietética direcionada a gestantes busca guiar os hábitos alimentares para a adequação da oferta, tanto de macro e micronutrientes, quanto de energia, cujas necessidades encontram-se aumentadas diante das demandas do processo gestacional ${ }^{3}$.

A literatura destaca que deficientes ingestões de micronutrientes podem estar relacionadas ao retardo de crescimento intrauterino, à pré-eclâmpsia, a anomalias congênitas e ao aborto espontâneo ${ }^{4}$.

Além disso, a evolução do peso gestacional possui relação direta com o crescimento fetal e com o peso ao nascer. $\mathrm{O}$ inadequado peso ao nascer é uma das grandes preocupações da saúde pública, em decorrência de sua associação à morbimortalidade durante o primeiro ano de vida e ao elevado risco da criança desenvolver doenças durante a vida adulta, tais como diabetes e obesidade, quando nasce macrossômica e síndrome metabólica, naquelas de baixo peso ${ }^{4}$.

A avaliação do consumo alimentar neste processo, auxilia a descoberta de hábitos desfavoráveis e da ingestão inadequada. A abordagem do consumo alimentar permite o planejamento de recomendações dietéticas baseadas em grupos de imentos ${ }^{5}$. 


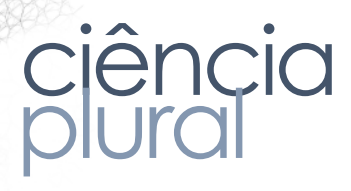

Além disso, a avaliação da ingestão de nutrientes por gestantes, ao longo do período gestacional, poderá auxiliar nas estratégias de promoção da saúde e da gestação saudável por profissionais da atenção primária6 .

Dessa forma, o objetivo do presente estudo foi investigar o consumo de nutrientes por gestantes adultas, assistidas na atenção básica de Palmas, Tocantins, buscando identificar precocemente desvios evitáveis por meio de orientações dietéticas pré-natais.

\section{Metodologia}

A rede de saúde de Palmas, no estado do Tocantins, encontra-se dividida em sete territórios de saúde, que representam as diferentes localidades geográficas. Cada território de saúde, conta com 3 a 6 centros de saúde da comunidade (CSC), conforme tamanho da população territorial.

O estudo investigou mulheres em quatro dos sete territórios de saúde de Palmas, sendo a amostra calculada e distribuída em função do número total de atendimentos pré-natais realizados nos CSC do território, conforme dados do SISPRENATAL.

Os quatro territórios de saúde escolhidos foram os que concentravam o maior número de acompanhamentos pré-natais e acompanharam 1581 mulheres no ano considerado. O território denominado Karajá, realizou 566 acompanhamentos, o território Krahô realizou 406; o território Apinajé acompanhou 317 pré-natais e o território Xambioá, 292.

A amostra foi calculada, aplicando-se a fórmula proposta por Cardoso ${ }^{7}$ :

$\mathrm{N}=[(\mathrm{Za}+\mathrm{Z} \beta) / \mathrm{C}] 2+3$

Onde: $\mathrm{N}=$ total de indivíduos necessários; $\mathrm{Z} \alpha=$ desvio-padrão para $\alpha ; \mathrm{Z} \beta=$ desvio-padrão para $\beta ; C=0,5 \times \ln [(1+r) /(1-r)]$, sendo $r=$ coeficiente de correlação perado e $\ln =$ logaritmo neperiano. 
Assim, a amostra foi composta por 50 gestantes, sendo 18 do território Karajá; 13 do território Krahô, 10 do território Apinajé e 9 do Xambioá, proporcionalmente ao número de pré-natais realizados nos territórios.

A partir de listas fornecidas pelas equipes de saúde, foram sorteadas a participar do estudo gestantes de idade superior a 18 anos, do primeiro, segundo e terceiro trimestres gestacionais. Foram consideradas elegíveis para participação as gestantes, residentes nos territórios de saúde estudados e usuárias da rede pública de saúde.

O questionário foi aplicado somente após a assinatura do Termo de Consentimento Livre e Esclarecido por parte das gestantes. A primeira parte do questionário comtemplou informações a respeito da participante e o período gestacional, bem como dados sociodemográficos como renda familiar, nível de instrução materna e do chefe familiar, ocupação no mercado de trabalho das mães investigadas, número de cômodos e aglomeração no domicílio.

A segunda parte foi a aplicação do Recordatório 24 horas que investigou o consumo alimentar nas 24 horas anteriores à entrevista, detalhando informações como: tipo de alimento consumido, horários, refeições consumidas, local de consumo, modo de preparo, porções consumidas e suas respectivas quantidades.

Os dados coletados de consumo alimentar tiveram as medidas caseiras traduzidas em gramas e mililitros e sua composição de nutrientes foi estimada pelo Dietbox, pesquisando-se os alimentos nas tabelas de composição química de alimentos, na seguinte ordem: pesquisou-se o alimento inicialmente na tabela proposta por Philippi ${ }^{8}$, seguida da Tabela de Composição de Alimentos da Unicamp (TACO) ${ }^{9}$ e, quando o alimento não foi localizado em nenhuma delas, utilizou-se a tabela do $\mathrm{IBGE}^{10}$.

Foram calculados os teores de ingestão diária de energia, carboidratos, roteínas, lipídios totais, fibras, cálcio, ferro, zinco, potássio, sódio, magnésio, fósforo, aminas A, C, D, E, B1, B2, B3, B6, B9, B12, por meio da dieta, sem considerar os rientes obtidos por meio da suplementação medicamentos. A adequação da stão, conforme a recomendação diária, foi calculada conforme proposto pelas 
Dietary Reference Intakes (DRI) ${ }^{11,12}$. Os desvios-padrão intrapessoais de micronutrientes, utilizados nos cálculos, foram os propostos por Marchioni, Slater e Fisberg13. Para o calciferol foi utilizado o desvio-padrão intrapessoal proposto por Morimoto et $\mathrm{al}^{14}$ para mulheres adultas.

O banco de dados foi construído utilizando-se o programa Microsoft Excel 365 e analisado no programa Statistical Package for Social Sciences versão 23.0 (SPSS).

Foi realizada uma análise descritiva utilizando-se frequências absoluta e relativa e medidas de tendência central com seus respectivos valores de dispersão, com objetivo de descrever o comportamento das principais variáveis avaliadas no estudo.

O presente estudo foi aprovado pelo Comitê de Ética em Pesquisa com seres humanos da Universidade Federal do Tocantins, sob o número de parecer: 2.702.535.

\section{Resultados e Discussão}

As tabelas 1 e 2 trazem as características sociodemográficas, de saúde e nutrição da população estudada.

Tabela 1: Características sociodemográficas de gestantes, da atenção básica no município de Palmas -TO, 2019. ( $n=50)$.

\begin{tabular}{lc}
\hline \multicolumn{1}{c}{ Variáveis } & Estatística \\
\hline Idade (anos) & $25,32 \pm 5,44^{\mathrm{a}}$ \\
\hline Escolaridade (anos de estudo formal) & $11,60 \pm 2,71^{\mathrm{a}}$ \\
\hline Renda domiciliar (em reais) & $1019,00(1341,95-2047,65)^{\mathrm{c}}$ \\
\hline Renda per capita (em reais) & $499,50(423,09-700,26)^{\mathrm{c}}$ \\
\hline Zona residencial (\%) & $80^{\mathrm{b}}$ \\
Urbana & $20^{\mathrm{b}}$ \\
Rural & $38^{\mathrm{b}}$ \\
\hline Trabalho remunerado (\%) & $62^{\mathrm{b}}$ \\
Sim & $3,00(3,07-4,17)^{\mathrm{c}}$ \\
Não & \\
\hline Número de pessoas no domicílio &
\end{tabular}


Tabela 2: Características de saúde e estado nutricional de gestantes, da atenção básica no município de Palmas -TO, 2019. $(n=50)$.

\begin{tabular}{|c|c|}
\hline Variáveis & Estatística \\
\hline Peso pré-gestacional & $63,19 \pm 14,82^{\mathrm{a}}$ \\
\hline Peso Atual & $68,31 \pm 15,45^{\mathrm{a}}$ \\
\hline \multicolumn{2}{|l|}{ Classificação do EN• pré-gestacional (\%) } \\
\hline Baixo peso & $6^{\mathrm{b}}$ \\
\hline Eutrofia & $54^{b}$ \\
\hline Sobrepeso & $24^{b}$ \\
\hline Obesidade & $16^{\mathrm{b}}$ \\
\hline \multicolumn{2}{|l|}{ Classificação do EN• atual (\%) } \\
\hline Baixo peso & $22^{\mathrm{b}}$ \\
\hline Eutrofia & $42^{b}$ \\
\hline Sobrepeso & $18^{\mathrm{b}}$ \\
\hline Obesidade & $18^{\mathrm{b}}$ \\
\hline Idade gestacional atual & $26,80 \pm 9,03^{a}$ \\
\hline Número de consultas pré-natais & $5,28 \pm 3,33^{\mathrm{a}}$ \\
\hline Adequação do consumo energético (\%) & - \\
\hline$\geq \mathrm{RDA}^{*}$ Adequado & $18^{\mathrm{b}}$ \\
\hline$<$ RDA $^{*}$ Inadequado & $82^{b}$ \\
\hline Adequação dos Carboidratos ao AMDR** (\%) & - \\
\hline Adequado & $54^{b}$ \\
\hline Inadequado & $46^{\mathrm{b}}$ \\
\hline \multicolumn{2}{|l|}{$\begin{array}{l}\text { Adequação das Proteínas ao recomendado por peso } \\
\text { corporal }(\%)\end{array}$} \\
\hline Adequado & $32^{b}$ \\
\hline Inadequado & $68^{\mathrm{b}}$ \\
\hline \multicolumn{2}{|l|}{ Adequação das Proteínas ao AMDR** (\%) } \\
\hline Adequado & $96^{\mathrm{b}}$ \\
\hline Inadequado & $04^{\mathrm{b}}$ \\
\hline \multicolumn{2}{|l|}{ Adequação dos Lipídeos ao AMDR** (\%) } \\
\hline Adequado & $54^{\mathrm{b}}$ \\
\hline Inadequado & $46^{\mathrm{b}}$ \\
\hline
\end{tabular}

Nota: a Médias e Desvios-padrão; ${ }^{\mathrm{b}}$ Percentuais; $\bullet$ EN: estado nutricional; ${ }^{*}$ RDA: Recommended Dietary Allowances -Ingestão Dietética Recomendada; ${ }^{* *}$ AMDR: Acceptable Macronutrient Distribution Ranges Intervalos Aceitáveis de Distribuição dos Macronutrientes.

Na Tabela 3 é possível observar os consumos diários de macro e micronutrientes pela população estudada, comparados às recomendações para a idade e ciclo da vida. Destacaram-se $82 \%$ das gestantes com ingestões inadequadas de energia e retinol; $98 \%$ de inadequação para o ácido fólico e 94\% para ferro, cálcio e cobalamina. A ingestão diária de calciferol esteve inadequada em 100\% das gestantes. 
Tabela 3: Consumos diários e adequações da ingestão de macro e micronutrientes por gestantes, da atenção básica no município de Palmas -TO, 2019 (n=50).

\begin{tabular}{|c|c|c|c|}
\hline Nutriente & Ingestão Diária & $\begin{array}{c}\text { Percentuais de } \\
\text { Inadequações da } \\
\text { Ingestão }\end{array}$ & $\begin{array}{c}\text { Classificação } \\
\text { da Ingestão Habitual }\end{array}$ \\
\hline Energia (Kcal) & $1930,50(1722,29-2203,22)^{b}$ & 82 & Insuficiente \\
\hline Carboidratos (g) & $199,15(209,55-278,66)^{b}$ & 46 & Insuficiente \\
\hline Proteínas (g) & $84,10 \pm 40,77^{a}$ & 68 & Insuficiente \\
\hline Lipídeos $(g)$ & $68,23(65,92-91,58)^{\mathrm{b}}$ & 46 & Insuficiente \\
\hline Fibras $(g)$ & $18,98 \pm 13,09 a$ & 81,6 & Insuficiente \\
\hline Cálcio $(m g)$ & $456,22(410,11-624,75)^{b}$ & 94 & Insuficiente \\
\hline Fósforo (mg) & $819,70(744,51-984,46)^{b}$ & 54 & Insuficiente \\
\hline Magnésio (mg) & $216,56 \pm 109,02^{a}$ & 92 & Insuficiente \\
\hline Ferro (mg) & $13,92(13,58-18,60)^{\mathrm{b}}$ & 94 & Insuficiente \\
\hline Zinco (mg) & $12,14 \pm 6,38^{a}$ & 76 & Insuficiente \\
\hline Sódio (mg) & $2116,40 \pm 988,45^{a}$ & 88 & Excessiva \\
\hline Potássio (mg) & $2343,46 \pm 1217,50^{a}$ & 88 & Insuficiente \\
\hline Retinol ( $\mu g$ ) & $296,85(386,20-3158,69)^{\mathrm{b}}$ & 82 & Insuficiente \\
\hline Calciferol $(\mu g)$ & $2800(4426-1,50)^{\mathrm{b}}$ & 100 & Insuficiente \\
\hline Tocoferol (mg) & $12,95(12,95-20,55)^{\mathrm{b}}$ & 62 & Insuficiente \\
\hline Ácido ascórbico (mg) & $68,20(24,83-1102,20)^{\mathrm{b}}$ & 62 & Insuficiente \\
\hline Tiamina $(m g)$ & $1,42 \pm 6947^{a}$ & 70 & Insuficiente \\
\hline Riboflavina (mg) & $1,43(1,30-2,38)^{\mathrm{b}}$ & 56 & Insuficiente \\
\hline Niacina (mg) & $22,04 \pm 12,37 \mathrm{a}$ & 58 & Insuficiente \\
\hline Piridoxina (mg) & $1,58(1,41-2,50)^{\mathrm{b}}$ & 66 & Insuficiente \\
\hline Folato $(\mu g)$ & $157,31(148,98-225,20)^{\mathrm{b}}$ & 98 & Insuficiente \\
\hline Cobalamina $(\mu g)$ & $4,29(1,52-29,61)^{b}$ & 94 & Insuficiente \\
\hline
\end{tabular}

Notas: a Médias \pm Desvios-padrão; ${ }^{b}$ Medianas e Intervalos de Confiança de 95\%.

No presente estudo observou-se alta prevalência de inadequação no consumo de energia. A literatura afirma que a gestação ocasiona alterações fisiológicas no organismo materno, que aumentam as necessidades energéticas ${ }^{15}$. $\mathrm{O}$ aporte energético inadequado à gestante pode desencadear competição entre a mãe e o feto, restringindo o crescimento fetal. São recomendados adicionais energéticos entre 340 e 440 Kcal, a partir do segundo e durante o terceiro trimestres, além das necessidades energéticas calculadas para a mulher adulta não grávida ${ }^{16}$.

Os principais objetivos do incremento das necessidades energéticas durante o período gestacional são: compensar os gastos associados à elevação do metabolismo aracterístico da gestação; promover o aumento de peso adequado na gestante para roporcionar adequado crescimento fetal, da placenta e de outros tecidos associados ândulas mamárias etc); garantir a energia adicional para atividade física e 
composição corporal materna durante o período gestacional; e garantir reservas essenciais para o período da lactação ${ }^{17}$.

No presente estudo $81,6 \%$ das gestantes apresentaram inadequado consumo de fibras. A literatura aponta que a constipação intestinal é uma adversidade digestiva bastante frequente, sendo muito comum no período gestacional ${ }^{18}$. A ingestão de $28 \mathrm{~g}$ de fibras diárias ou de 14 g para cada 1000 Kcal ingeridas é o recomendado na gestação e contribui para evitar a constipação. A gestante deve ser orientada para o consumo de verduras e legumes, grãos integrais, frutas, aumentando a ingestão hídrica ${ }^{19}$.

Outro destaque foi para a elevada prevalência de inadequação do consumo de cálcio na população e gestantes aqui estudada. Para a literatura, especialmente durante o terceiro trimestre, quando dentes e ossos do bebê estão se formando e mineralizando, as gestantes devem receber aporte de cálcio extra. $\mathrm{O}$ corpo da gestante nesse período também estoca cálcio para a produção de leite materno no pós-parto. Quando a alimentação materna é deficiente nesse mineral, podem surgir cáries dentárias, câimbras nas pernas, perda dentária, além de osteoporose no futuro. Quando a calcemia materna se mantém baixa, as reservas de cálcio do organismo feminino são mobilizadas para o suprimento das necessidades fetais ${ }^{20}$.

Em nosso estudo, chamou a atenção o elevado percentual de mulheres (94\%) com ingestões insuficientes de magnésio (94\%), ferro (94\%) e ácido fólico (98\%). Estudo realizado no Hospital Universitário da Universidade de São Paulo, relacionou a deficiência do magnésio a complicações durante a gestação, como restrição de crescimento intrauterino, pré-eclâmpsia, eclâmpsia e maiores taxas de mortalidade fetal $^{21}$. A anemia por deficiência de ferro está associada ao baixo peso ao nascer, à prematuridade, à maior incidência de doenças infecciosas, ao menor desenvolvimento físico e neurológico de recém-nascidos, e ao aumento da mortalidade perinatal 22. Como parte da assistência no pré-natal, a suplementação de ácido fólico e ferro durante a gravidez é recomendada para diminuir o risco de anemia por deficiência de ferro na gestante e baixo peso ao nascer da criança ${ }^{23}$. A deficiência de ácido fólico urante a gestação pode acarretar sérios prejuízos ao desenvolvimento embrionário, ando associada à ocorrência dos defeitos do tubo neural (DTN), além de predispor 
também a lábio leporino, abortos espontâneos, doença cardíaca congênita, teratogenicidade e pré-eclâmpsia ${ }^{24}$.

Também muito prevalentes na população de gestantes estudadas foram as inadequações de ingestão de retinol e de cobalamina. A deficiência de retinol na gestação está relacionada à ocorrência das síndromes hipertensivas da gravidez, à prematuridade, à anemia gestacional, à sepse puerperal, ao estresse oxidativo e ao maior risco de aborto espontâneo, que colaboram para a elevação das taxas de mortalidade materna e de recém-nascidos ${ }^{25}$. Em estudo que avaliou a anemia megaloblástica e seus efeitos fisiopatológicos, a deficiência de cobalamina em gestantes pode causar anemia megaloblástica, caracterizada por diversos defeitos na síntese do DNA, culminando em um conjunto de anormalidades da medula óssea e hematológicas.

No que se refere aos eletrólitos, $88 \%$ das gestantes apresentaram ingestões inadequadas de sódio e de potássio. Considerando-se a importância desses dois eletrólitos na regulação da pressão arterial, sua inadequação na dieta de mulheres grávidas pode ocasionar edema, alterações nos níveis pressóricos maternos e no desenvolvimento fetal ${ }^{3,4}$.

Conhecer o consumo alimentar da gestante é uma estratégia importante que permite estabelecer intervenções individualizadas, em momentos oportunos, conforme as necessidades de cada paciente.

Destaca-se a importância de uma abordagem individualizada e detalhada das gestantes pela equipe de saúde, para proporcionar cuidados que garantam uma gestação mais saudável e segura para a mãe e o bebê.

Segundo Marchioni ${ }^{5}$ o método do recordatório alimentar possui limitações, o entrevistado deve recordar, definir e quantificar a ingestão alimentar do dia anterior ao da entrevista e/ou a ingestão pode ser omitida pelo subregistro. Além disso, no caso específico de gestantes, sabe-se que a alteração do estado fisiológico e psicológico, nuitas vezes pode influenciar os resultados de estudos de análise do consumo mentar. No presente estudo foi aplicado um único recordatório alimentar de 24 
horas, o que pode não ter refletido exatamente a ingestão habitual de nutrientes das participantes.

\section{Conclusão}

O grupo de gestantes estudado está ingerindo nutrientes essenciais ao período gestacional, aquém do recomendado, estando o binômio mãe-filho sujeitos a resultados obstétricos indesejáveis, caso nenhuma intervenção seja realizada.

Destaca-se a importância da assistência pré-natal de qualidade, com identificação precoce das inadequações do estado nutricional em gestantes e a implementação de intervenções eficazes e em tempo oportuno.

\section{Referências}

1. Bertin LR, Parisenti J, Di Pietro FP, Vasconcelos GAF. Métodos de avaliação do consumo alimentar de gestantes: uma revisão. Rev Bras Saúde Matern Infant. 2006; 6(4):383-390.

2. Bang SW, Lee SS. The factors affecting pregnancy outcomes in the second trimester pregnant women. Nutr Res Pract. 2009;3(2):134-140.

3. Saunders C. Síndromes hipertensivas da gravidez - SHG. In: Accioly E, Saunders C, Lacerda EMA. Nutrição em obstetrícia e pediatria. $2^{a}$ ed. Rio de Janeiro: Cultura Médica; 2002. p. 189-207.

4. Rodrigues GH, Freitas CJ, Freitas SVL, Sena LCK. Consumo de sódio e potássio por gestantes do Vale do Jequitinhonha. Ciência e Saúde. 2017;10(1):39-47.

5. Marchioni DML, Gorgulho BM, Steluti J. Consumo Alimentar: guia para avaliação. $1^{a}$ ed. São Paulo: Manole, 2019. 300p.

6. Castro TBM, Souza GAR, Vilela FAA, Kac G. Association between sociodemographics factors and dietary patterns during pregnancy. Rev. Nutr. 2014; 27(2):173-181.

7. Cardoso MA. Desenvolvimento, validação e aplicações de questionários de frequência alimentar em estudos epidemiológicos. Kac G, Sichieri R, Gigante DP, organizadores. Epidemiologia nutricional. Rio de Janeiro: Editora Fiocruz/São Paulo: Atheneu, p. 201-12, 2007. 
8. Philippi ST. Tabela de Composição de Alimentos Suporte para Decisão Nutricional. 3ed. São Paulo: Manole; 2013.

9. Universidade Estadual de Campinas - Unicamp. Tabela brasileira de composição de alimentos - TACO. 4ed. Campinas: Unicamp/NEPA; 2011.

10. Brasil. Ministério do Planejamento, Orçamento e Gestão. Instituto Brasileiro de Geografia e Estatística. Diretoria de Pesquisas. Coordenação de Trabalho e Rendimento. Pesquisa de orçamentos familiares 2008-2009 - Tabelas de Composição Nutricional dos Alimentos Consumidos no Brasil. Rio de Janeiro: IBGE; 2011.

11. Cuppari LM. Aplicações das DRI's na avaliação da ingestão de nutrientes para indivíduos. In: International Life Sciences Institute do Brasil. Usos e Aplicações das “Dietary Reference Intakes”, DRIs. São Paulo: ILSI Brasil; 2001. p. 22-34.

12. Cominetti C, Cozzolino SMF. Recomendações de Nutrientes. São Paulo: International Life Sciences Institute do Brasil; 2017.

13. Marchioni DML, Slater B, Fisberg RM. Aplicação das Dietary Reference Intakes na avaliação da ingestão de nutrientes para indivíduos. Rev.Nutr. 2004;17(2):207-216.

14. Morimoro MJ, Marchioni LMD, Cesar GLC, Fisberg MR. Within-person variance for adjusting nutrient distribution in epidemiological studies. Rev. Saúde Pública. 2011;45(3):1-4.

15. Freitas SE, Bosco DMS, Sippel AC, Lazzaretti KR. Recomendações nutricionais na gestação. Destaques acadêmicos. 2010;2(3):81-95.

16. Teixeira D, Pestana D, Calhau C, Vicente L, Graça P. Alimentação e nutrição na gravidez; 2015[acesso em 22 nov 2019]. Disponível em: https:/ / www.alimentacaosaudavel.dgs.pt/activeapp/wpcontent/files_mf/1444899925Alimentacaoenutricaonagravidez.pdf

17. Butte N, King J. Energy Requirements During Pregnancy and Lactation. Public Health Nutrition. 2005;8(7);1010-1027.

18. Williams SR. Nutrição durante a gravidez e lactação. In: Williams SR. Fundamentos de nutrição e dietoterapia. 6a ed. Porto Alegre: Artes Médicas; 2001.

19. Institute of medicine. Dietary reference intakes for energy, carbohydrate, fiber, fat, fattu acids, cholesterol, protein and amino acids (macronutrients). Washington: IOM; 2005. 
20. Affonso VC, Sonati GJ. Nutrição e o ciclo da Vida: gravidez, amamentação e a criança Pré-escolar. In: Vilarta R. Alimentação Saudável, Atividade Física e Qualidade de Vida. Campinas: IPES editorial; 2007. p. 35-45.

21. Rocha SV. Avaliação bioquímica e do consumo alimentar de magnésio em mulheres saudáveis no terceiro trimestre gestacional [dissertação]. São Paulo: Universidade de São Paulo; 2009. 97 p.

22. Schümann K, Ettle T, Szegner B, Elsenhans B, Solomons NW. On risks and benefits of iron supplementation recommendations for iron intake revisited. J Trace Elem Med Biol.2007; 21(3):147-168.

23. World Health Organization. Guideline: daily iron and folic acid supplementation in pregnant women. Geneva: WHO; 2012.

24. Wang J, Trudinger BJ, Duarte N. Elevate circulating homocysteine levels in placental vascular disease and associated pre-eclampsia. $\mathrm{Br} \mathrm{J}$ of Obstet Gynaecol. 2000;107(7):935-938.

25. Eigbefoh JO, Okpere EE, Ande B, Asonye C. How useful is the Helen Keller food frequency chart in the determination of the vitamin A status in pregnancy? J Obstet Gynaecol. 2005; 25(2):123-127. 